\title{
Avaliação da procura pelos serviços de saúde e tratamento farmacológico de pacientes com anorexia e bulimia nervosa
}

Evaluation of the search for health services and pharmacological treatment of patients with anorexia and bulimia nervosa Evaluación de la búsqueda por los servicios de salud y tratamiento farmacológico de pacientes con anorexia y bulimia nerviosa

\author{
Fernando Yamamoto CHIBA \\ Suzely Adas Saliba MOIMAZ \\ Artênio José Isper GARBIN \\ Cléa Adas Saliba GARBIN
}

Departamento de Odontologia Infantil e Social, Universidade Estadual Paulista (UNESP), Faculdade de Odontologia de Araçatuba 16015-050 Araçatuba-SP, Brasil

\begin{abstract}
Resumo
Introdução: Os transtornos alimentares são caracterizados como distúrbios do comportamento alimentar, associados ao desequilíbrio nos pensamentos, ações e atitudes dos indivíduos resultando em prejuízos à saúde do indivíduo. Estas condições são cada vez mais comuns na sociedade atual e têm ganhado crescente atenção da comunidade científica. Objetivo: Analisar a procura pelo atendimento e farmacoterapia em mulheres com anorexia e bulimia nervosa atendidas em uma faculdade de medicina em 2018. Material e método: Realizou-se análise documental dos prontuários médicos. A procura pelo atendimento foi considerada não-espontânea quando a paciente foi encaminhada pela unidade de urgência/emergência ou compareceu acompanhada por responsável legal sem admitir necessidade de tratamento. Resultados: Identificou-se 14 pacientes, com idade média de 31,21 anos. 43\% apresentaram procura não-espontânea pelo atendimento, sendo $83 \%$ destas encaminhadas por unidades de urgência/emergência. Foram prescritos 21 medicamentos diferentes, sendo a maioria antidepressivos. 52\% dos fármacos prescritos não são disponibilizados pelo Sistema Único de Saúde. 29\% dos pacientes apresentavam polifarmácia, 43\% automedicação e 57\% pensamento de morte. Houve associação entre o pensamento de morte e uso de 4 ou mais medicamentos. Conclusão: Uma parcela considerável das pacientes teve procura não-espontânea pelo atendimento. Os fármacos prescritos foram principalmente antidepressivos e a maioria não é disponibilizado no Sistema Único de Saúde, evidenciando a onerosidade econômica e social do tratamento. Descritores: Transtornos da Alimentação e da Ingestão de Alimentos; Anorexia; Bulimia; Tratamento Farmacológico.
\end{abstract}

\begin{abstract}
Introduction: Eating disorders are characterized as disorders of eating behavior, associated with imbalance in the thoughts, actions and attitudes of individuals resulting in harm to the health of the individual. These conditions are increasingly common in today's society and have gained increasing attention from the scientific community. Objective: To analyze the search for health services and pharmacotherapy in women with anorexia and bulimia nervosa attended at a medical school in 2018. Material and method: Documental analysis of medical records was performed. The search for care was considered non-spontaneous when the patient was referred by the emergency unit or accompanied by a legal guardian without admitting need for treatment. Results: We identified 14 patients with a mean age of 31.21 years. $43 \%$ showed non-spontaneous search for care, which $83 \%$ were referred by emergency units. 21 different drugs were prescribed, most of them antidepressants. 52\% of the drugs prescribed are not available from Brazilian Unified Health System. $29 \%$ of the patients presented polypharmacy, $43 \%$ self-medication and 57\% thought death. There was an association between the thought of death and the use of 4 or more medications. Conclusion: A considerable number of patients had non-spontaneous search for care. The drugs prescribed were mainly antidepressants and most are not available in the Brazilian Unified Health System, evidencing the economic and social onerosity of the treatment.
\end{abstract}

Descriptors: Feeding and Eating Disorders; Anorexia; Bulimia; Drug Therapy.

\section{Resumen}

Introducción: Los trastornos alimentarios se caracterizan como disturbios del comportamiento alimentario, asociados al desequilibrio en los pensamientos, acciones y actitudes de los individuos resultando en perjuicios a la salud del individuo. Estas condiciones son cada vez más comunes en la sociedad actual y han ganado creciente atención de la comunidad científica. Objetivo: Analizar búsqueda de atención médica y farmacoterapia en mujeres con anorexia y bulimia nerviosa atendidas en una Facultad de medicina en 2018. Material y método: se realizó análisis documental de registros médicos. La búsqueda de atención médica fue considerada no espontánea cuando la paciente fue remitida por urgencia/emergencia o acompañada por responsable legal sin admitir necesidad de tratamiento. Resultados: se identificaron 14 pacientes, edad media de 31,21 años. $43 \%$ presentaron búsqueda no espontánea, $83 \%$ de estas remitidas por unidades de urgencia/emergencia. Fueron prescritos 21 medicamentos diferentes, la mayoría antidepresivos. 52\% de medicamentos prescritos no son proporcionados por el Sistema Único de Salud Brasileño. 29\% de los pacientes presentan polifarmacia, 43\% automedicación y 57\% pensamiento de muerte. Hubo asociación entre pensamiento de muerte y uso de 4 o más medicamentos. Conclusión: Parte considerable de los pacientes tuvo búsqueda no espontánea de atención médica. Los medicamentos prescritos fueron principalmente antidepresivos, la mayoría no disponible en el Sistema Único de Salud Brasileño, evidenciando la onerosidad económica y social del tratamiento.

Descriptores: Trastornos de Alimentación y de la Ingestión de Alimentos; Anorexia; Bulimia; Quimioterapia.

\section{INTRODUÇÃO}

Os transtornos alimentares são condições cada vez mais comuns e presentes na sociedade atual, de modo que o desenvolvimento de estudos que visam melhorar as ações e estratégias de tratamento e prevenção têm ganhado crescente atenção da comunidade científica nas últimas décadas ${ }^{1}$. Estes transtornos são caracterizados como distúrbios do comportamento alimentar, associados ao desequilíbrio nos pensamentos, ações e atitudes dos indivíduos acometidos, resultando em severos prejuízos físicos e psicológicos ${ }^{2}$.

Os cuidados envolvidos no tratamento de pessoas com transtornos alimentares envolvem elevados níveis de estresse e sobrecarga psicológica para os pacientes e seus familiares ${ }^{3}$. Tais condições estão entre as que apresentam os índices mais altos de mortalidade dentre os transtornos psiquiátricos, representando um severo problema de saúde pública ${ }^{4}$.

A etiologia dos transtornos alimentares não é totalmente elucidada, porém sabe-se que a origem é multifatorial, envolvendo fatores genéticos, epigenéticos e ambientais, constituindo-se em um distúrbio biopsicossocial ${ }^{5}$. Desse modo, é possível sugerir que a interação entre o comportamento do indivíduo, o meio em que se desenvolve e as experiências de vida acumuladas pode determinar o 
desenvolvimento de um transtorno alimentar no contexto individual ${ }^{6}$.

Os transtornos alimentares mais conhecidos, anorexia e bulimia nervosa, são caracterizados por comportamentos alimentares extremos, medo de engordar, desejo persistente de emagrecer, sobrevalorização do peso e da forma com acentuada distorção da imagem corporal ${ }^{7}$. A prevalência dessas condições é tipicamente maior em mulheres jovens e de alta renda, possivelmente devido a fatores culturais e econômicos, entretanto, tem ocorrido o aumento do número de casos em diversos países ${ }^{8}$.

A anorexia nervosa é caracterizada pela apresentação de um peso corporal significativamente baixo para a estatura, idade e estágio de desenvolvimento do indivíduo que não está relacionado a outra condição de saúde ou à indisponibilidade de alimentos. $\mathrm{O}$ baixo peso corporal é associado a um padrão persistente de comportamentos para prevenir o restabelecimento do peso normal, que pode incluir extrema restrição alimentar, atos de purgação e aumento do gasto energético, associado ao medo de ganho de peso ${ }^{9}$.

A bulimia nervosa é caracterizada por episódios recorrentes e frequentes de compulsão alimentar nos quais o indivíduo experimenta uma perda do controle sobre o comportamento alimentar, comendo notavelmente mais e se sentindo incapaz de parar ou limitar o tipo e a quantidade de comida ingerida. A compulsão alimentar é acompanhada por repetidos comportamentos compensatórios inadequados, tais como atos de purgação, destinados a evitar o ganho de peso. $O$ indivíduo exibe preocupação excessiva com a forma e peso do corpo, influenciando sua autoavaliação. Não há perda significativa de peso e, portanto, diferencia-se da anorexia nervosa?

O tratamento do paciente envolve a integração de ações de uma equipe multidisciplinar e o uso constante de fármacos de uso controlado, representando um desafio à administração de recursos financeiros, materiais e humanos do Sistema Único de Saúde (SUS) ${ }^{10,11}$. A prevenção e o controle dos agravos nutricionais requerem um conjunto amplo de medidas de diversos setores, visando organizar, qualificar e aperfeiçoar as ações de enfrentamento da complexidade da situação alimentar e nutricional da população. Assim, a Política Nacional de Alimentação e Nutrição reconhece que os cuidados aos indivíduos com transtornos alimentares fazem parte do escopo da atenção nutricional no SUS ${ }^{12}$.

O objetivo deste estudo foi analisar a procura pelo serviço de saúde e a farmacoterapia em mulheres diagnosticadas com anorexia e bulimia nervosa atendidas em um Ambulatório Especializado de Saúde Mental de uma Faculdade pública brasileira de medicina, no ano de 2018.

\section{MATERIAL E MÉTODO}

O estudo foi aprovado pelo Comitê de Ética em Pesquisa (CAAE: 80497417.1.0000.5420) e conduzido de acordo com as normas constantes nas Resoluções do Conselho Nacional de Saúde ${ }^{\circ}$ 466, de 12 de dezembro de 2012; e n ${ }^{\circ} 510$, de 7 de abril de 2016. Todas as pacientes assinaram o Termo de Consentimento Livre e Esclarecido contendo o detalhamento sobre os objetivos da pesquisa e a forma de divulgação dos dados presentes em seus prontuários. Salienta-se que a assinatura do responsável legal foi obtida nos casos de pacientes com idade inferior a 18 anos.

Foi realizada a análise documental dos prontuários médicos de pacientes diagnosticados com transtorno alimentar, em tratamento no Ambulatório Especializado de Saúde Mental de uma Faculdade pública de medicina, no ano de 2018.

Para a composição da amostra deste estudo foram selecionados os prontuários dos pacientes que realizaram o tratamento no período estudado e atenderam aos critérios de elegibilidade: diagnóstico de anorexia ou bulimia nervosa, ser do sexo feminino e não ter abandonado o tratamento. Desta forma, prontuários de 14 mulheres constituíram a amostra deste estudo, a partir dos quais realizou-se uma análise retrospectiva para investigar os dados referentes a idade, relato de pensamento de morte, procura pelo serviço de saúde, fator considerado desencadeador do transtorno alimentar, tipo e quantidade de medicamentos prescritos $\mathrm{e}$ automedicação.

A procura pelo serviço foi considerada nãoespontânea nos casos em que a paciente foi encaminhada pela unidade de urgência/emergência ou quando compareceu acompanhada por responsável legal sem admitir a necessidade de tratamento.

A disponibilidade dos medicamentos prescritos pelo SUS foi analisada utilizando-se a Relação Nacional de Medicamentos Essenciais (RENAME). ${ }^{13}$

Os dados foram processados utilizando o programa Epi Info versão 7.2. A análise foi realizada por meio de estatística descritiva e o teste Exato de Fisher, adotando nível de significância de $5 \%$, foi utilizado para verificar a existência de associação entre as variáveis relato de pensamento de morte, quantidade de medicamentos prescritos e a procura pelo serviço de saúde.

\section{RESULTADOS}

Foram analisados 14 prontuários médicos de pacientes do sexo feminino, com idade média de $31,21 \pm 12,98$ anos de idade. Conforme observado na tabela 1, houve casos de pacientes em diferentes faixas etárias, desde indivíduos com idade inferior aos 18 anos até acima dos 40. Uma parcela considerável das pacientes apresentou procura não 
espontânea pelo serviço de saúde, sendo $83 \%$ destas encaminhadas por unidades de urgência e emergência (Tabela 1).

Tabela 1. Distribuição absoluta e percentual das pacientes diagnosticadas com anorexia e bulimia, segundo a idade, pensamento de morte e procura pelo serviço de saúde

\begin{tabular}{|c|c|c|}
\hline Variável & $\mathbf{n}$ & $\%$ \\
\hline $\begin{array}{l}\text { Faixa etária (anos) } \\
<18 \\
18 \mathrm{I}-\mathrm{I} 30 \\
31 \mathrm{I}-\mathrm{I} 40 \\
>40 \\
\text { Total }\end{array}$ & $\begin{array}{r}4 \\
3 \\
3 \\
4 \\
\mathbf{1 4}\end{array}$ & $\begin{array}{r}28,57 \\
21,43 \\
21,43 \\
28,57 \\
\mathbf{1 0 0 , 0 0}\end{array}$ \\
\hline $\begin{array}{l}\text { Relato de pensamento de morte } \\
\text { Sim } \\
\text { Não } \\
\text { Total }\end{array}$ & $\begin{array}{r}8 \\
6 \\
14\end{array}$ & $\begin{array}{r}57,14 \\
42,86 \\
\mathbf{1 0 0 , 0 0}\end{array}$ \\
\hline $\begin{array}{l}\text { Procura pelo serviço de saúde } \\
\text { Espontâneo } \\
\text { Não-espontâneo } \\
\text { Total }\end{array}$ & $\begin{array}{r}8 \\
6 \\
\mathbf{1 4}\end{array}$ & $\begin{array}{r}57,14 \\
42,86 \\
\mathbf{1 0 0 , 0 0}\end{array}$ \\
\hline $\begin{array}{l}\text { Pacientes com procura não espontânea } \\
\text { Encaminhamento pela urgência/emergência } \\
\text { Acompanhado por responsável legal sem admitir } \\
\text { necessidade de tratamento } \\
\text { Total }\end{array}$ & $\begin{array}{l}1 \\
6 \\
\end{array}$ & $\begin{array}{r}83,33 \\
16,67 \\
\mathbf{1 0 0 , 0 0} \\
\end{array}$ \\
\hline
\end{tabular}

A maioria das mulheres apresentou relato de pensamento de morte (Tabela 1) e dentre os fatores considerados desencadeadores do transtorno alimentar destacaram-se as dificuldades financeiras e problemas conjugais (Figura 1).

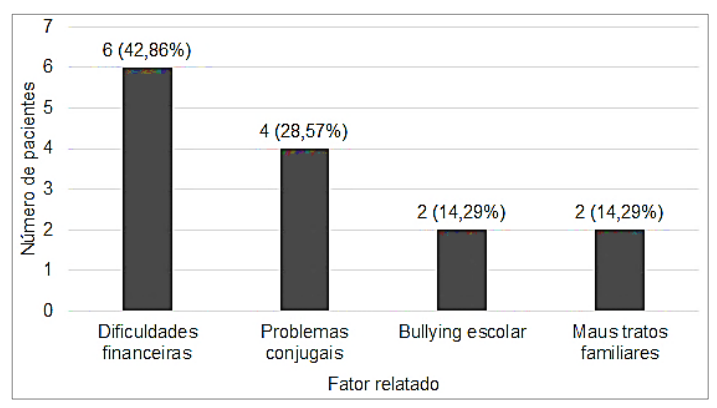

Figura 1: Distribuição absoluta e percentual do fator considerado desencadeador do transtorno alimentar, segundo relato das pacientes com anorexia e bulimia nervosa.

A tabela 2 demonstra que foram constatadas prescrições de 21 tipos de medicamentos diferentes durante o tratamento dos indivíduos, com predomínio de antidepressivos e ansiolíticos. Observou-se a ocorrência de polifarmácia em $28,57 \%$ dos pacientes, salientando que a maioria dos fármacos prescritos não era disponibilizada pelo SUS (Tabela 2). A prática de automedicação foi observada em $43 \%$ dos casos e estava relacionada ao consumo de laxativos, purgantes e diuréticos.

Tabela 2. Características da farmacoterapia utilizada pelas pacientes com anorexia e bulimia nervosa

\begin{tabular}{lrr}
\hline \multicolumn{1}{c}{ Variável } & $\mathbf{n}$ & $\mathbf{\%}$ \\
\hline Tipo de medicamento prescrito & & \\
Antidepressivo & 9 & 42,86 \\
Ansiolitico & 4 & 19,05 \\
Antipsicótico & 3 & 14,29 \\
Anticonvulsivante & 2 & 9,52 \\
Tratamento de insônia & 1 & 4,76 \\
Regulação do apetite & 1 & 4,76 \\
Controle da secreção ácida estomacal & 1 & 4,76 \\
Total & $\mathbf{2 1}$ & $\mathbf{1 0 0 , 0 0}$ \\
Quantidade de medicamentos prescritos por paciente & & \\
1 & & 21,43 \\
2 & 3 & 7,14 \\
3 & 1 & 14,29 \\
4 & 2 & 28,57 \\
5 ou mais & 4 & 28,57 \\
Total & 4 & $\mathbf{1 0 0}, \mathbf{0 0}$ \\
Disponibilidade dos medicamentos prescritos pelo SUS & $\mathbf{1 4}$ & \\
Sim & 10 & 47,62 \\
Não & 11 & 52,38 \\
Total & $\mathbf{2 1}$ & $\mathbf{1 0 0 , 0 0}$ \\
\hline
\end{tabular}

Como visto na tabela 3, houve associação estatisticamente significante $(\mathrm{p}<0,05)$ entre o relato de pensamento de morte e a prescrição de 4 ou mais medicamentos.

Tabela 3. Associação entre o relato de pensamento de morte, quantidade de medicamentos prescritos por paciente e procura pelo serviço de saúde em pacientes com anorexia e bulimia nervosa.

\begin{tabular}{|c|c|c|c|c|c|c|c|}
\hline \multirow{2}{*}{ Variáveis } & \multicolumn{7}{|c|}{ Relato de pensamento de morte } \\
\hline & & Sim & & Não & & Total & $\begin{array}{c}\text { p- } \\
\text { valor }\end{array}$ \\
\hline $\begin{array}{l}\text { Quantidade de medicamentos } \\
\text { prescritos por paciente }\end{array}$ & $\mathbf{n}$ & $\%$ & $\mathbf{n}$ & $\%$ & $\mathbf{n}$ & $\%$ & \\
\hline$<4$ & 1 & 12,50 & 5 & 83,33 & 6 & 42,86 & \\
\hline 4 ou mais & 7 & 87,50 & 1 & 16,67 & 8 & 57,14 & $0,0256^{*}$ \\
\hline Total & 8 & 100,00 & 6 & 100,00 & 14 & 100,00 & \\
\hline Procura pelo serviço de saúde & $\mathbf{n}$ & $\%$ & $\mathbf{n}$ & $\%$ & $\mathbf{n}$ & $\%$ & \\
\hline Espontâneo & 5 & 62,50 & 3 & 50,00 & 8 & 57,14 & \\
\hline Nã̃o espontâneo & 3 & 37,50 & 3 & 50,00 & 6 & 42,86 & 1,0000 \\
\hline Total & 8 & 100,00 & 6 & 100,00 & 14 & 100,00 & \\
\hline
\end{tabular}

\section{DISCUSSÃO}

A ocorrência de anorexia e bulimia nervosa é comumente associada com mulheres jovens. Embora estudos prospectivos indiquem que $\mathrm{o}$ final da adolescência e o início da idade adulta seja o período de maior desenvolvimento dos transtornos alimentares, é possível que essas condições se manifestem mais tardiamente para alguns indivíduos $^{14,15}$. Interessantemente, observou-se neste estudo uma ampla variação na faixa etária das pacientes, incluindo indivíduos com idade inferior aos 18 anos até acima dos 40 . Verificou-se que 50\% das pacientes apresentava idade superior aos 30 anos, evidenciando a importância do desenvolvimento de estudos que investiguem o surgimento dos transtornos alimentares em mulheres após a idade adulta jovem, principalmente considerando que as estratégias voltadas para a prevenção e o tratamento desses distúrbios podem apresentar eficácia variável dependendo do estágio da vida do indivíduo.

Observou-se que $43 \%$ das pacientes apresentou procura não espontânea ao Ambulatório Especializado de Saúde Mental, sendo a maioria destas encaminhadas por unidades de urgência e emergência, sugerindo que muitas vezes o início do tratamento ocorre somente quando o indivíduo se encontra em estágios avançados do transtorno alimentar, com comprometimento severo das condições de saúde. Neste sentido, estudos demonstram que os indivíduos acometidos pela anorexia e bulimia nervosa tendem a negar a necessidade de tratamento, esconder sua condição e evitar a ajuda profissional, o que pode resultar no agravamento das complicações decorrentes dos distúrbios $^{8,16}$. Tais fatos dificultam a realização e diminuem a eficácia de pesquisas epidemiológicas de modo que os estudos geralmente utilizam registros médicos de hospitais em uma área circunscrita, o que pode subestimar a ocorrência dos transtornos alimentares na população em geral, pois nem todos os pacientes são diagnosticados ou encaminhados para atendimento em hospitais ou centros de saúde mental $^{8,17}$. 
O presente estudo revelou que o pensamento de morte foi relatado por $57,14 \%$ das mulheres. Esta preocupante condição é corroborada por estudos clínicos nos quais confirmou-se que indivíduos com distúrbios alimentares frequentemente apresentam também uma série de distúrbios psiquiátricos adicionais ${ }^{18,19}$. O transtorno alimentar associado com as comorbidades depressão e transtorno de ansiedade trata-se da variante clínica mais comumente observada do distúrbio, exercendo importante influência sobre à gravidade dos sintomas psicológicos e risco de suicídio ${ }^{18}$. Nesse contexto, sabe-se que o atendimento multidisciplinar é fundamental no tratamento dos transtornos alimentares, considerando as significativas sequelas médicas e psicológicas associadas a essas condições $^{11,20}$. Além do restabelecimento nutricional e terapia psiquiátrica, medicamentos psicotrópicos podem ser prescritos para tratar o transtorno alimentar e suas comorbidades psiquiátricas ${ }^{20}$. De fato, no presente estudo, observou-se que os principais medicamentos prescritos para o tratamento das pacientes eram antidepressivos $(42,86 \%)$ e ansiolíticos (19,05\%).

A análise da farmacoterapia utilizada pelas pacientes demonstrou que a maioria delas $(57,14 \%)$ utilizava quatro ou mais medicamentos simultaneamente. Estes achados estão de acordo com um estudo longitudinal que acompanhou o tratamento de pacientes com anorexia nervosa ao longo de 13 anos e verificou um aumento significativo de pacientes que apresentavam polifarmácia ${ }^{21}$. Esses dados são preocupantes, considerando os efeitos adversos conhecidos $\mathrm{e}$ as possíveis interações medicamentosas dos fármacos utilizados no tratamento dos transtornos alimentares.

A Relação Nacional de Medicamentos Essenciais (RENAME) foi elaborada com o intuito de servir como instrumento para promover o uso racional e orientar o financiamento de medicamentos na assistência farmacêutica ${ }^{13}$. A partir de sua análise, constatou-se que a maioria (52,38\%) dos medicamentos prescritos para o tratamento das pacientes com anorexia e bulimia nervosa não eram disponibilizados pelo SUS. A administração dos recursos voltados para a assistência farmacêutica representa um grande desafio para os gestores do SUS, diante da complexidade das necessidades de saúde da população, da velocidade da inovação científico-tecnológica e dos diferentes modelos de organização e financiamento do sistema de saúde ${ }^{13}$. A baixa disponibilidade dos medicamentos pode prejudicar a continuidade dos tratamentos instituídos, podendo consequentemente elevar os custos com a atenção à saúde e comprometer a qualidade e eficácia das ações terapêuticas ${ }^{22}$. Assim, a deficiência na disponibilidade dos medicamentos somada à relutância dos indivíduos em aderir ao tratamento pode influenciar de modo determinante no prognóstico do paciente com transtorno alimentar ${ }^{23}$.

Convém salientar que embora neste estudo o principal fator relatado como desencadeador do transtorno alimentar tenha sido a dificuldade financeira, estudos indicam que a origem dos transtornos alimentares é multifatorial, com influências genéticas e ambientais, tais como fatores socioculturais que incluem exposição à mídia que apresenta padrões do corpo ideal, lojas que por vezes oferecem produtos voltados apenas para pessoas altas e magras, além de extensiva propaganda da indústria da dieta ${ }^{5,24,25}$. Estas influências puderam ser notadas neste estudo, visto que a prática de automedicação foi observada em $43 \%$ dos casos e relacionava-se ao consumo de laxativos, purgantes e diuréticos. As limitações deste estudo incluem o fato de que as participantes eram mulheres atendidas em um Ambulatório Especializado de Saúde Mental de uma Faculdade pública brasileira de medicina e, portanto, representam um perfil específico de pacientes. É possível sugerir que isto tenha influenciado o tamanho da amostra, pois além da tendência dos pacientes de não procurar atendimento, estudos relatam maior ocorrência da anorexia e bulimia nervosa em pacientes com alta renda ${ }^{8}$. Nesse sentido, este estudo desperta a necessidade do desenvolvimento de ações e estratégias voltadas para um novo perfil de população-alvo.

Achados fundamentais deste estudo foram as informações de que o relato de pensamento de morte foi significativamente associado ao uso simultâneo de 4 ou mais medicamentos, que a dificuldade financeira foi o principal fator relatado como desencadeador do transtorno alimentar e que a maioria dos medicamentos prescritos não é disponibilizado pelo SUS. Tomados em conjunto, estes dados revelam a situação de vulnerabilidade a que estas pacientes estão expostas, pois sugere que indivíduos em condições de extrema fragilidade psicológica podem ter dificuldade ou não conseguir acesso à farmacoterapia indicada para seu tratamento, justamente em função do fator relatado como causador do transtorno alimentar. Portanto, torna-se fundamental a realização de uma avaliação precoce, precisa e criteriosa, considerando as condições do paciente, visando o tratamento dos transtornos alimentares e a prevenção da evolução de comorbidades psiquiátricas graves.

\section{CONCLUSÃO}

Com base nos dados obtidos, conclui-se que uma parcela considerável das pacientes com anorexia e bulimia nervosa apresentou procura não espontânea aos serviços de saúde.

Os fármacos prescritos durante o tratamento foram principalmente antidepressivos, destacando-se o fato de que a maioria não é disponibilizado no SUS, 
o que evidencia a onerosidade econômica e social do tratamento.

\section{AGRADECIMENTOS}

Os autores agradecem à Coordenação de Aperfeiçoamento de Pessoal de Nível Superior (CAPES) pelo auxílio financeiro para a realização do estudo.

\section{REFERÊNCIAS}

1. Le LK, Barendregt JJ, Hay P, Mihalopoulos C. Prevention of eating disorders: A systematic review and meta-analysis. Clin Psychol Rev. 2017;53:46-58.

2. Herpertz-Dahlmann B. Adolescent eating disorders: definitions, symptomatology, epidemiology and comorbidity. Child Adolesc Psychiatr Clin N Am. 2009;18(1):31-47.

3. Zabala MJ, Macdonald P, Treasure J. Appraisal of caregiving burden, expressed emotion and psychological distress in families of people with eating disorders: a systematic review. Eur Eat Disord Rev. 2009;17(5):338-49.

4. Sharan P, Sundar AS. Eating disorders in women. Indian J Psychiatry. 2015; 57(Suppl 2): S286S295.

5. Brandys MK, de Kovel CG, Kas MJ, van Elburg AA, Adan RA. Overview of genetic research in anorexia nervosa: The past, the present and the future. Int J Eat Disord. 2015;48(7):814-25.

6. Mitchison D, Hay PJ. The epidemiology of eating disorders: genetic, environmental, and societal factors. Clin Epidemiol. 2014;6:89-97.

7. American Psychiatric Association; 2013. American Psychiatric Association: Diagnostic and Statistical Manual of Mental Disorders. 5th ed. Arlington.

8. Smink FR, van Hoeken D, Hoek HW. Epidemiology of eating disorders: incidence, prevalence and mortality rates. Curr Psychiatry Rep. 2012;14(4):406-14.

9. Geneva: World Health Organization; 1992. World Health Organization. The ICD-10 classification of mental and behavioural disorders. Clinical descriptions and diagnostic guidelines.

10. Stewart TM, Williamson DA. Multidisciplinary treatment of eating disorders--Part 1: Structure and costs of treatment. Behav Modif. 2004;28(6):812-30.

11.Donaldson AA, Hall A, Neukirch J, Kasper V, Simones S, Gagnon S, et al. Multidisciplinary care considerations for gender nonconforming adolescents with eating disorders: A case series. Int J Eat Disord. 2018;51(5):475-479.

12.Brasil. Ministério da Saúde. Secretaria de Atenção à Saúde. Departamento de Atenção Básica. Política Nacional de Alimentação e Nutrição/Ministério da Saúde, Secretaria de
Atenção à Saúde. Departamento de Atenção Básica. Brasília: Ministério da Saúde, 2013. 84 p.

13.Brasil. Ministério da Saúde. Secretaria de Ciência, Tecnologia e Insumos Estratégicos. departamento de assistência farmacêutica e insumos estratégicos. Relação nacional de medicamentos essenciais: RENAME 2017. Brasília: Ministério da Saúde, 2017. 210 p.

14. Stice E, Marti CN, Rohde P. Prevalence, incidence, impairment, and course of the proposed DSM-5 eating disorder diagnoses in an 8-year prospective community study of young women. $\mathbf{J}$ Abnorm Psychol. 2013;122(2):445-57.

15.Lewinsohn PM, Striegel-Moore RH, Seeley JR. Epidemiology and natural course of eating disorders in young women from adolescence to young adulthood. J Am Acad Child Adolesc Psychiatry. 2000;39(10):1284-92.

16.van Son GE, van Hoeken D, Bartelds AI, van Furth EF, Hoek HW. Time trends in the incidence of eating disorders: a primary care study in the Netherlands. Int J Eat Disord. 2006;39(7):565-9.

17. Hoek HW, van Hoeken D. Review of the prevalence and incidence of eating disorders. Int $\mathbf{J}$ Eat Disord. 2003;34(4):383-96.

18. Brand-Gothelf A, Leor S, Apter A, Fennig S. The impact of comorbid depressive and anxiety disorders on severity of anorexia nervosa in adolescent girls. J Nerv Ment Dis. 2014;202(10):759-62.

19.Bühren K, Schwarte R, Fluck F, Timmesfeld N, Krei M, Egberts K, et al. Comorbid psychiatric disorders in female adolescents with first-onset anorexia nervosa. Eur Eat Disord Rev. 2014;22(1):39-44.

20.Mizusaki K, Gih D, LaRosa C, Richmond R, Rienecke RD. Psychotropic usage by patients presenting to an academic eating disorders program. Eat Weight Disord. 2018 Jun 7. doi: 10.1007/s40519-018-0520-3. [Epub ahead of print]

21.Fazeli PK, Calder GL, Miller KK, Misra M, Lawson EA, Meenaghan E, et al. Psychotropic medication use in anorexia nervosa between 1997 and 2009. Int J Eat Disord. 2012;45(8):970-6.

22.Nascimento RCRM, Álvares J, Guerra Junior AA, Gomes IC, Costa EA, Leite SN et al. Availability of essential medicines in primary health care of the Brazilian Unified Health System. Rev. Saúde Pública. 2017;51(Suppl 2):10s.

23.Fassino S, Abbate-Daga G. Resistance to treatment in eating disorders: a critical challenge. BMC Psychiatry. 2013;13:282.

24.Becker AE, Fay KE, Agnew-Blais J, Khan AN, Striegel-Moore RH, Gilman SE. Social network media exposure and adolescent eating pathology in Fiji. Br J Psychiatry. 2011;198(1):43-50. 
25.Groesz LM, Levine MP, Murnen SK. The effect of experimental presentation of thin media images on body satisfaction: a meta-analytic review. Int $\mathbf{J}$ Eat Disord. 2002;31(1):1-16.

\section{CONFLITO DE INTERESSES}

Os autores declaram não haver conflitos de interesse.

\section{AUTOR PARA CORRESPONDENCIA}

\section{Fernando Yamamoto Chiba}

fernando.chiba@unesp.br

Submetido em 12/12/2018

Aceito em 12/03/2019 